Research Article

\title{
Chitosan Biomedical Applications for the Treatment of Viral Disease: A Data Mining Model Using Bibliometric Predictive Intelligence
}

\author{
Worasak Klongthong $\mathbb{D}^{1},{ }^{1}$ Veera Muangsin ${ }^{D}{ }^{2}{ }^{2}$ Chupun Gowanit, \\ and Nongnuj Muangsin $\mathbb{1}^{3}$ \\ ${ }^{1}$ Technopreneurship and Innovation Management, Graduate School, Chulalongkorn University, Bangkok, Thailand \\ ${ }^{2}$ Department of Computer Engineering, Faculty of Engineering, Chulalongkorn University, Bangkok, Thailand \\ ${ }^{3}$ Department of Chemistry, Faculty of Science, Chulalongkorn University, Bangkok, Thailand \\ Correspondence should be addressed to Nongnuj Muangsin; nongnuj.j@chula.ac.th
}

Received 13 October 2020; Revised 24 November 2020; Accepted 3 December 2020; Published 28 December 2020

Academic Editor: Massimiliano F. Peana

Copyright (c) 2020 Worasak Klongthong et al. This is an open access article distributed under the Creative Commons Attribution License, which permits unrestricted use, distribution, and reproduction in any medium, provided the original work is properly cited.

Chitosan has attracted increasing attention from researchers in the pharmaceutical and biomedical fields as a potential agent for the prevention and treatment of infectious diseases. However, identifying the development of emerging technologies related to this biopolymer is difficult, especially for newcomers trying to understand the research streams. In this work, we designed and implemented a research process based on a bibliometric predictive intelligence model. Our aim is to glean detailed scientific and technological trends through an analysis of publications that include certain word phrases and related research areas. Cross correlation, factor mapping, and the calculation of "emergent" scores were also used. A total of 1,612 scientific papers on chitosan technology related to viral disease treatment published between 2010 and 2020 were retrieved from the Web of Science. Results from the keyword modelling quantitatively highlight three major frontier research and development topic groups: drug delivery and adjuvants, vaccines and immune response, and tissue engineering. More specifically, the emergent scores show that much of the chitosan-based treatment for viral diseases is in the in vitro stage of development. Most chitosan applications are in pharmacology/pharmacy and immunology. All results were confirmed by experts in the field, which indicates that the validated process can be applied to other fields of interest.

\section{Introduction}

In recent years, emergent viral diseases have become a global challenge for public healthcare systems [1]. Even older viruses such as influenza are capable of re-emerging and presenting new epidemic and pandemic threats. Woolhouse and Gaunt [2] estimated that there are 1,399 species of human pathogen, among which 87 were first reported in humans after 1980, and they are responsible for much of the mortality, morbidity, and massive economic losses worldwide. Viruses follow different strategies to infect humans-some do so directly, whereas others spread through animals serving as reservoir hosts. During the past four decades, the World Health Organization has documented multiple disease outbreaks in different regions due to viral agents such as Ebola,
Crimean-Congo hemorrhagic fever virus, Lassa virus, Rift Valley fever virus, Marburg virus, Middle East respiratory syndrome-related coronavirus, Nipah virus, and severe acute respiratory syndrome coronaviruses [3]. In December 2019, Wuhan, China, became the epicenter of the most recent coronavirus (SARS-CoV-2) outbreak, which has since spread internationally [4]. In 2020, the World Health Organization declared the disease, novel coronavirus (COVID-19), to be a global health emergency [5]. Countering the impacts of viral disease outbreaks requires research and development (R\&D) collaboration among global institutions. Current vaccination approaches include the use of lentogenic live-virus vaccines or inactivated vaccines to induce protective immunity while producing minimal adverse effects in chickens and other birds [6]. 
Drug delivery is a field of pharmaceutical sciences in which various treatment strategies are developed for a range of ailments, and drug delivery research is opening up new avenues for improving therapies. There are multiple types of carrier systems and a variety of routes through which drugs can be administered to the body. For instance, conventional carrier systems include tablets and capsules, and novel carrier systems include technologies such as nanoparticles and microspheres [7]. In recent years, there has been a major emphasis on integrating different types of polymers into carrier systems, and scientists linked with the medical and pharmaceutical fields have carried out extensive research to harness their potential in the field of drug delivery and carrier systems [8].

Although a variety of polymers are deployed in drug delivery applications, priority is given to those that are natural, biosafe, and biodegradable. Natural biopolymers are produced by a variety of microorganisms and biomaterials such as collagen, chitin, silk, and starch [9]. Biopolymers are primarily distinguishable from fossil-fuel-derived polymers because of their immunogenic behavior, which endows them with a unique ability to degrade when exposed to natural enzymes, thereby enhancing their sustainability. As such, biophysical, biochemical, and biomedical researchers in various fields have intensified their efforts toward the development of biomaterials using natural polymers.

Chitin and its derivative, chitosan, are biopolymers of crustacean origin and are similar in structure to cellulose. They are amino polysaccharides with amide/amino functionality and hydroxyl groups that can undergo chemical modifications to yield a variety of materials for applications in biomedicine, healthcare, biotechnology, manufacturing, the food industry, and agrochemicals, among others [10-12]. Chitosan is commercially produced by the deacetylation of chitin, which is the structural element in crustacean exoskeletons (e.g., crabs and shrimp) and fungal cell walls. Chitosan is soluble in dilute aqueous acidic solutions but insoluble in water and organic solvents. Shrimp shells, which contain about $25 \%-40 \%$ chitin, are the main source of chitosan, whereas crab shells contain approximately $15 \%-$ $20 \%$ chitin. Chitosan is nontoxic, stable, biodegradable, and sterilizable. Moreover, it is mucoadhesive because of its electrostatic interactions with mucosal surfaces [13]. Its reactivity and polycationic character enable the production of a variety of formulations with different properties, ranging from hydrogels, rods, and fibers to nano-/microparticles and membranes [14]. Altogether, these properties make chitosan a versatile material with a high number potential applications in the biomedical and biotechnological fields [15].

The development of drug delivery systems is among the best-known biomedical applications of medical-grade chitosan. The amino and carboxyl groups interact with glycoprotein in mucus to form a hydrogen bond in the chitosan molecule, thereby producing an adhesive effect. Because mucoprotein is positively charged, chitosan and mucus are attracted to each other, which improves drug bioavailability and prolongs in vivo retention and release time [16]. Various formulation methods can be used to prepare chitosan nanoparticles to release an active ingredient (such as proteins, peptides, or DNA vaccines) in a sustained manner over a prolonged period. Chitosan can be easily functionalized to enhance its adsorption capacity, and ionic crosslinking for the preparation of chitosan nanoparticles containing proteins, peptides, and vaccines has received significant attention in recent years. This is because of the simplicity of the processes, which involve no chemical cross linkers, organic solvents, or high temperatures [17]. Chitosan has been approved as GRAS (Generally Recognized as Safe) by the United States Food and Drug Administration, and the Mexican government's relaxation of previously stringent regulations has resulted in the development of new and large-scale pharmaceutical and biomedical manufacturing facilities in that region. Thus, there are extensive opportunities to use chitosan in the biomedical industry, and the biomedical applications of chitosan have proliferated as a result of extensive research activities.

Chitosan nanoparticles (CNPs) have a plethora of biological applications in drug delivery. Various types of chitosan-made drug carriers have been conceived for oral, sublingual, nasal, transdermal, parenteral, vaginal, cervical, intrauterine, and rectal administration routes, among others. A recent market analysis of Grand View Research (2020) estimated the value of the global chitosan market at USD 6.8 billion in 2019, and it is projected to expand at a revenue-based compound annual growth rate of $24.7 \%$ between 2020 and 2027 [18]. The growing application of chitosan products in water treatment, as well as the pharmaceutical, biomedical, cosmetics, and food industries, is expected to further drive market growth.

Hundreds of studies related to drug delivery systems using chitosan have been published [19, 20]. However, trends in studies related to the use of chitosan to treat viral disease are not well understood because of the wide variety of research themes. To our knowledge, a comprehensive overview of chitosan research related to viral disease or biomedical aspects has not been published. Accordingly, in this research, we propose a novel bibliometric approach to track trends in the development of research on chitosan in viral disease treatment as a test case. We further discover emergent trends and technologies using bibliometric analysis.

One objective of this research is to understand current trends and predict upcoming innovations, as well as identify frontier R\&D trends for future technologies. Another objective is to illustrate a technique for creating review articles automatically that produces accurate predictions. This approach is faster than the conventional approach to creating review articles, which is slow and requires a large amount of energy to summarize hundreds of papers on a topic of interest.

Moreover, the results obtained from the analysis were confirmed by experts in this field, which demonstrates that the developed process will be valid for other topics as well. The benefits will be realized by researchers, research institutes, and private companies, as well as wider society. For instance, new researchers who are interested in this field can understand the overall research streams of chitosan-related 
viral disease treatments. Furthermore, current researchers can understand the overall research and map it to their expertise to explore the "white space" or gaps in the research of the future.

\section{Methodology and Data}

2.1. Emergent Technologies. Like "convergence," the concept of "emergence" is "widely used but seldom defined" [21], which is likely because the term is used in diverse ways [22]. Goldstein [23] characterized emergence as comprising radical novelty and coherence or correlation. Moreover, it is dynamical and perceivable. The properties most commonly associated with emergence across all definitions are novelty and growth. Emerging technologies (ETs) can be defined as technologies in a nascent phase of development or those that will be developed during the next five to ten years and are projected to have a substantial socioeconomic impact. Halaweh [24] proposed that all technologies that are not used in a specific context may be considered ET, given their lack of a limited lifetime. Consequently, ETs are caused by disruptive transformations in the fields where human beings are central to development. Emerging technology research is attracting increasing attention from academic institutions and industry because of its potential as an indicator for commercial innovation, as well as breakthrough science and technology. At the microlevel, ET research seeks practical measures to recognize "hot" R\&D topics and, thereby, contribute to $\mathrm{R} \& \mathrm{D}$ policy, the analysis of technological opportunities, and innovation management to foster the creation of new industries or the transformation of existing ones [25-28].

Forecasting ETs is full of challenges. Prediction has been demonstrated to be an effective tool for anticipating and understanding the potential patterns, pace, and impacts of technological change [27]; however, it is more difficult to forecast the emergence of new technologies. Publications and patent data are major sources for the operationalization of ETs [29]. Emergent topics can be found in many sources that range from expert opinions [30] to bibliometric analyses of patents or R\&D publications [31]. Cozzens et al. [21] classified emergent technology forecasting methods into two main categories: (1) searches for rapidly increasing publication numbers in an existing field or topic and (2) data mining, which entails identifying patterns of emergence through the co-occurrence analysis of keywords (cokeywords) and citations (cocitations). Bibliometric analysis is a quantitative data mining method that complements expert assessments of scientific and technological emergence $[32,33]$. Two main directions predominate bibliometric data mining: (1) identifying existing ETs [34] and (2) predictive analysis that recognizes new technologies prior to their actual emergence [35]. For example, Upham and Small [36] generated cocitations using the ISI database from 1999 to 2004 to identify the most prolific emergent science and technology research topics, and Porter et al. [37] implemented an algorithm to calculate emergence scores from topical keywords in abstract records. The authors further presented the steps for generating $\mathrm{R} \& \mathrm{D}$ indicators to highlight "hot topic" terms and key players in their development.

2.2. Attributes of Emergence. High emergent scores provide a sense of frontier R\&D topics of interest, as well as organizational strategic directions and industry opportunities in a given domain. To identify emergent topics related to chitosan and viral diseases, our research model consolidates four key attributes of technological emergence: (1) impact on society, i.e., whether the ET will potentially benefit a wide range of economic and/or social sectors [38]; (2) newness or innovativeness with "the potential to create a new industry or transform existing ones" [39]; (3) growth, i.e., increasing $\mathrm{R} \& \mathrm{D}$ outputs and ongoing development $[40,41]$; and (4) the involvement of communities of practice, implying that multiple players, not all within a single research unit or entity, are collaborating in some manner, as indicated by, e.g., citation links in R\&D literature analyses [42].

\subsection{Text Mining and Natural Language Processing (NLP).} This study uses natural language processing (NLP) to extract key phrases from article titles, keywords, and abstracts in order to obtain the terms of emerging topics related to chitosan in viral disease research. In text mining, previously unknown and difficult-to-reveal information is derived from text data and then transformed into numeric data that can be used in data mining algorithms $[43,44]$. NLP techniques incorporate computational linguistics, artificial intelligence, mathematics, and information science to enable computers to understand natural language and perform particular tasks [45]. NLP expands the capabilities of text mining, as it can be utilized to analyze the semantic and grammatical structures of text. NLP algorithms have been applied in medicine, biochemistry, and other fields [46]. Various NLP methods include part-of-speech tagging, tokenization, splitting, parsing, and text chunking, i.e., grouping adjacent words in a sentence [47]. Porter et al. [37] used NLP and text mining techniques to calculate emergence scores for topical terms by merging title and abstract record sets from scientific papers. Their approach revealed several emergent topics, including nanoenabled drug delivery, dye-sensitized solar cells, and nonlinear programming.

2.4. Research Process. In this study, a bibliometric predictive intelligence model adapted from the approach in $[48,49]$ was used to identify emergent technology (Figure 1).

This bibliometric-based model measures emergence by tabulating and tracking patterns in the R\&D literature. Text mining tools are able to extract useful intelligence from science, technology, and innovation resources [50]. Technological trends can be traced by analyzing the text of topical content to consolidate important terms and phrases relating to a given concept or theme [51,52]. Those terms and phrases can then be tracked over time to glean evolutionary pathways [53].

The first step is to retrieve a set of research related to chitosan in scientific publication records from the Web of 


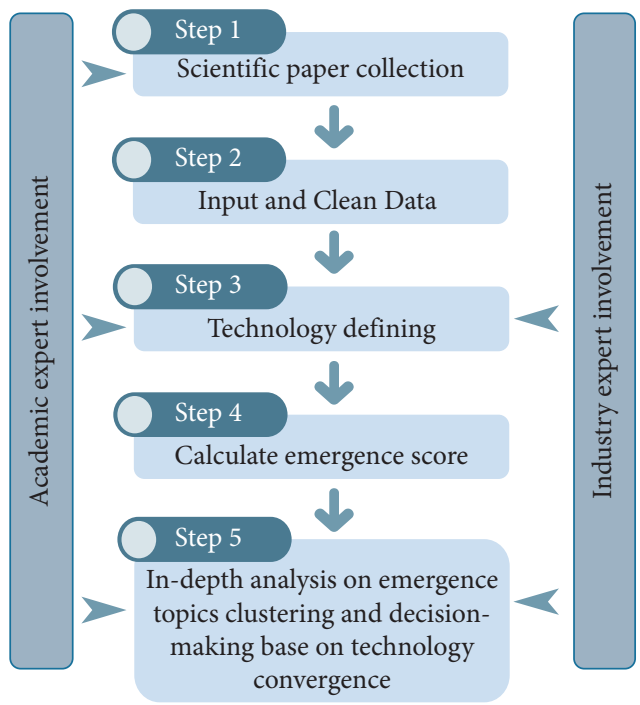

"Interested Area" from WoS

1. Import into VantagePoint

2. Cleaning data

1. Trend analysis

2. Academic impact analysis

3. Keyword analysis

1. Title (NLP)

2. Abstract (NLP)

3. Keywords

1. Emergence topic ranked by emergence score

2. Emergence topic grouping

FIGURE 1: Research process for identifying chitosan applications.

Science (WoS) database using several queries suggested by academic and medical experts. A total of 1,612 scientific papers published from 2010 to 2020 were retrieved from the database. All articles were subjected to text mining using VantagePoint $12.0 / \mathrm{PRO}$ to extract keyword patterns or relationships and identify trends and emergent topics related to chitosan applications in viral disease research. Next, the data were cleaned and the keywords and phrases from titles and abstracts were merged. Third, the preliminary patterns identified by the software were interpreted and defined by analyzing the trends and keywords; the outcomes were further analyzed by researchers, academic experts, and medical experts. Fourth, we calculated emergence scores from the keywords and phrases, for which we developed a custom "emergence indicator" script using VantagePoint software. The criteria used in the script were adapted from [37] and [49] and are as follows: (a) the term appears in the records for, at least, three years; (b) the term appears in seven or more records; (c) the ratio of records containing the term in the active period to those in the base period is, at least, $2: 1$; (d) the term cannot appear in $15 \%$ or more of the records from the base period; and (e) the term has more than one author that does not share the same record set.

These criteria target the four attributes of emergence (enduring societal impact, newness/novelty, growth, and community). Criteria (a) and (b) aim to assure a level of societal impact in that the topic has not occurred only in one or two years before disappearing. Moreover, these criteria indicate whether the topic relates to a "hot" global issue (e.g., a virus outbreak). In contrast, criteria (c) and (d) capture newness and growth because the term increasingly appears later in the study period. Criterion (e) ensures that research communities comprising multiple authors from multiple institutions have engaged with the topic. The specific levels were chosen based on experimentation with the test datasets in this study along with several others. However, the script enables users to vary the criteria thresholds so that the requirement of, at least, seven total records containing the term can be reduced for small datasets.

We use the formula based on the work of Wang et al. [49] to present exactly how the criteria in our VantagePoint script are computed. The process starts with a script term, the $t$ term, which refers to a continuous ten-year period in which the numbers 1 to 10 , respectively, denote one of those ten year units. Terms $t-1$ to $t-3$ were defined as the base period ( $\left.t_{\text {base }}\right)$, and $t-4$ to $t-10$ were defined as the active period $\left(t_{\text {active }}\right)$. Assuming a term $i$, its related parameters are described as follows.

The value of $n_{i t}$ is the number of records containing term $i$ in time $t$, which is expressed as

$$
x= \begin{cases}1, & \text { if term } i \text { appears in time period } t \\ 0, & \text { otherwise }\end{cases}
$$

We also have $N t$, which is the number of records in time period $t_{\text {active }}$ that include the set of trending keywords and phrases (title and abstract), for which we use term $i$. It is expressed as

$$
\begin{aligned}
|A| & =m, \\
A & =\left\{a_{1}, \ldots, a_{m}\right\}, \quad 1 \leq j \leq m, 1 \leq k \leq m, j \neq k, \\
y_{j k} & = \begin{cases}0, & a_{j} \text { and } a_{k} \text { co author one record, } \\
1, & \text { otherwise. }\end{cases}
\end{aligned}
$$

2.4.1. Enduring Societal Impact. A term must appear in, at least, one record during three-year-long periods and in, at least, seven total records in order to have the specified 
enduring societal impact attribute. This is expressed as follows:

$$
\text { if } \begin{aligned}
\sum_{t} x_{i t} & \geq 3, \\
\sum_{t} n_{i t} & \geq 7 .
\end{aligned}
$$

2.4.2. Newness. The term cannot appear in $15 \%$ or more of the base period records to have the specified newness attribute, which is expressed as follows:

$$
\text { if } \frac{\sum_{t=1}^{3} n_{i t}}{\sum_{t=1}^{10} n_{i t}} \leq 0.15
$$

2.4.3. Growth. The ratio of records containing the term during the active period to those in the base period must be, at least, $2: 1$ in order for it to have the specified growth attribute as follows:

$$
\text { if } \sum_{t=4}^{10} n_{i t} \geq 2 \times \sum_{t=1}^{3} n_{i t} .
$$

2.4.4. Community. The term needs to be used by more than one author who are not co-authors on the same set of records in order for term $i$ to have the specified community attribute as follows:

$$
\begin{aligned}
m & \geq 2, \\
\sum_{j=1}^{m} \sum_{k \neq j} y_{j k} & \geq 1 .
\end{aligned}
$$

Based on the equation given above, we apply the following equation, based on Porter et al. [37], to calculate the emergence score (EScore):

EScore $=2 \times$ Active Period Trend + Recent Trend + Slope

For a given term with seven periods of active data (the default), the calculation of the EScore for term $i$ is

$$
\begin{aligned}
\text { Active Trend }_{i} & =\left(\sum_{t=5}^{7} \frac{n_{i t}}{\sqrt{N_{t}}}-\sum_{t=1}^{3} \frac{n_{i t}}{\sqrt{N_{t}}}\right), \\
\text { Recent Trend }_{i} & =\left(\sum_{t=6}^{7} \frac{n_{i t}}{\sqrt{N_{t}}}-\sum_{t=4}^{5} \frac{n_{i t}}{\sqrt{N_{t}}}\right) \times 10, \\
\text { Slope }_{i} & =\frac{\left(n_{i 10} / \sqrt{N_{10}}\right)-\left(n_{i 7} / \sqrt{N_{7}}\right)}{3} \times 10, \\
\text { EScore }_{i} & =2 \times \text { Active Trend }_{i}+\text { Recent Trend }_{i}+\text { Slope }_{i} .
\end{aligned}
$$

As noted above, to identify emergent topics related to chitosan applications from 2010 to 2019, we used a 10-year test period comprising a 3-year base period followed by a 7year active period. We examined the resulting term scores

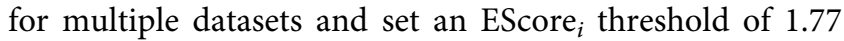
over which a term is considered emergent. Empirical observations indicate that a reasonable threshold will fall between 1.5 and 2 , and we selected 1.77 because it is the square root of $\pi$.

Finally, upcoming technological innovations were assessed based on trend analysis and emergent topics, which were ranked by their emergence scores. The technology convergence results were interpreted by academic and industry experts to ensure alignment with current and recent trends. A cross-correlation map was created by comparing emergent topics with WoS categories, and a factor map was created using principle component analysis (PCA), an unsupervised learning method similar to clustering that simplifies high-dimensional data while retaining trends and patterns by transforming them into fewer dimensions. Those dimensions then act as summaries of features [54, 55]. A common occurrence in biology, high-dimensional data arise when multiple features, such as the expression of many genes, are measured per sample. PCA finds patterns unbiased by prior knowledge about whether the samples are derived from distinct treatment groups or have phenotypic differences.

\section{Results and Discussion}

3.1. Annual Tally of Scientific Papers. Trend analysis of the 1,612 papers extracted from WoS in the final dataset revealed highly stable development in the target topic each year from 2010 to 2017 and more intensive development from 2018 onward. Figure 2 shows that, while an average of 150 papers on chitosan related to viral disease were published annually from 2010 to 2017 , that number increased by $20 \%$ from 2018 to 2019 , with a peak of 184 papers in 2018 . Overall research development also appears quite stable; however, as the inset of Figure 2 reveals, there was an exponential increase in terms related to immune responses and vaccines, including a $400 \%$ increase from 2017 to 2019 . This substantial increase in publications during the last few years reflects the intensification of research in this sector, which in turn illustrates the potential of technological goals to stimulate socially responsible research. These results demonstrate that chitosan-related technologies have become the subject of growing attention. Moreover, researchers are increasingly frequently investigating this topic, which could lead to research on developing chitosan applications in response to viral outbreaks.

3.2. Distribution of Publication Output. Examining the range of journals responsible for publishing the most frequently cited articles in different scientific fields is a useful exercise for tracking emergent research. Bradford's law states that most published papers in a given subject or field are concentrated in only few academic journals. Table 1 lists the top five journals that published work related to chitosan and viral diseases during the study period. They can be regarded as the core 


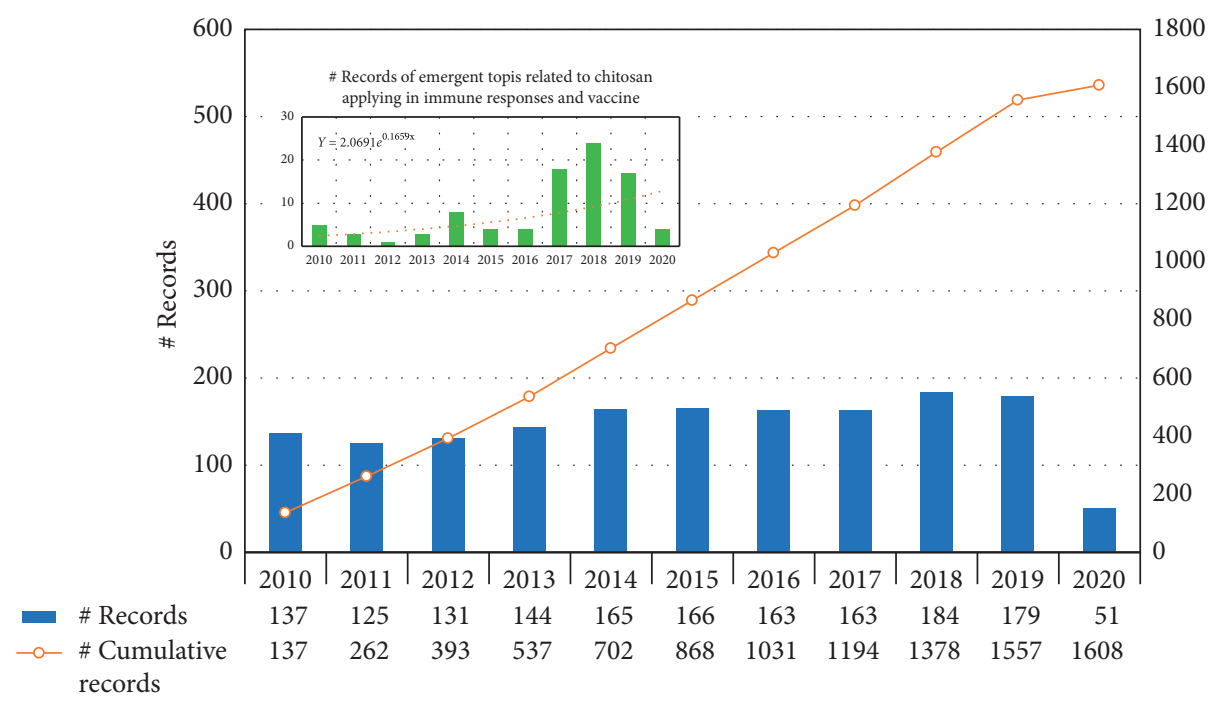

FIGURE 2: Annual distribution of papers on chitosan applications in viral disease treatment.

TABLE 1: Top five journal publishing in the field of chitosan research.

\begin{tabular}{lccc}
\hline Journal & $\begin{array}{c}\text { No. of } \\
\text { articles }\end{array}$ & Subject categories & $\begin{array}{c}\text { H } \\
\text { index }\end{array}$ \\
\hline Carbohydrate polymers & 62 & $\begin{array}{c}\text { Organic chemistry, materials, chemistry, polymers, and plastics } \\
\text { Agricultural and biological sciences (miscellaneous), biochemistry, genetics and } \\
\text { molecular biology (miscellaneous), and medicine (miscellaneous) }\end{array}$ \\
$\begin{array}{l}\text { Plos one } \\
\begin{array}{l}\text { International journal of } \\
\text { pharmaceutics }\end{array}\end{array}$ & 39 & Pharmacology, toxicology and pharmaceutics, and pharmaceutical science & 190 \\
$\begin{array}{l}\text { Journal of controlled release } \\
\text { International journal of biological } \\
\text { macromolecules }\end{array}$ & 35 & Pharmacology, toxicology and pharmaceutics, and pharmaceutical science & 237 \\
\hline
\end{tabular}

periodicals focused on this topic. We can see that Carbohydrate Polymers, which has an H index 172 (Q1), is the preeminent periodical in this field, having published $62(3.8 \%)$ related papers, followed by PLOS ONE (H index 268; Q1), which published 39 papers $(2.4 \%)$. In addition, the International Journal of Pharmaceutics, Journal of Controlled Release, and International Journal of Biological Macromolecules account for $2.4 \%, 2.2 \%$, and $1.6 \%$ of the total volume of published papers, respectively. The top five articles comprise three groups of subject studies, namely, polymers/plastics, molecular genetics, and pharmacology.

3.3. Research Hotspots. Research hotspots for chitosan applications were explored by analyzing high-frequency keywords. In general, keywords tend to both summarize the contents of a research article and refine the most essential concepts related to the main topic. Table 2 shows the top five keywords in chitosan-related viral disease papers identified through bibliometric analysis, namely, "adjuvant," "drug delivery," "gene delivery," "vaccine," and "tissue engineering," which account for $1.89 \%, 1.31 \%, 1.27 \%, 1.20 \%$, and $0.69 \%$ of the total 2,800 keywords, respectively. The top three keywords exhibit stable development; however, the fourthranked "vaccine" has received considerably more focus since 2018, indicating that this topic is emerging as a significant research focus.
The top five keywords and top five journals represent the research frontier areas of topic development. Adjuvants, drug delivery, and gene delivery are grouped into group 1: drug delivery development. As a natural polysaccharide extracted from the shells of arthropods such as crabs, shrimps, and lobsters, chitosan is a promising drug delivery vector for therapeutics and diagnostics because of its biocompatibility, low toxicity, biodegradability, and structural variability [56]. As the research shows, chitosan has a cationic character because of the primary amino groups, which are responsible for properties such as mucoadhesion, controlled drug release, transfection, in situ gelation, permeation enhancement, and efflux pump inhibitory properties [57]. Group 2 comprises vaccine development using chitosan. Kole et al. [58] encapsulated the inactivated VHSV vaccine with CNPs (CNPs-IV) by water-in-oil emulsification and demonstrated that immunization with CNPs-IV vaccines can organize a coordinative immune response. Group 3 encompasses tissue engineering applications. Croisier and Jérôme [59] studied the applications of chitosan as a polysaccharide in the field of tissue engineering. Their research includes the preparation and properties of chitosan-based biomaterials and the use of chitosan in two- and three-dimensional scaffolds and fibers, with a special focus on wound healing applications. 
TABle 2: Top five keywords in chitosan-related viral disease research.

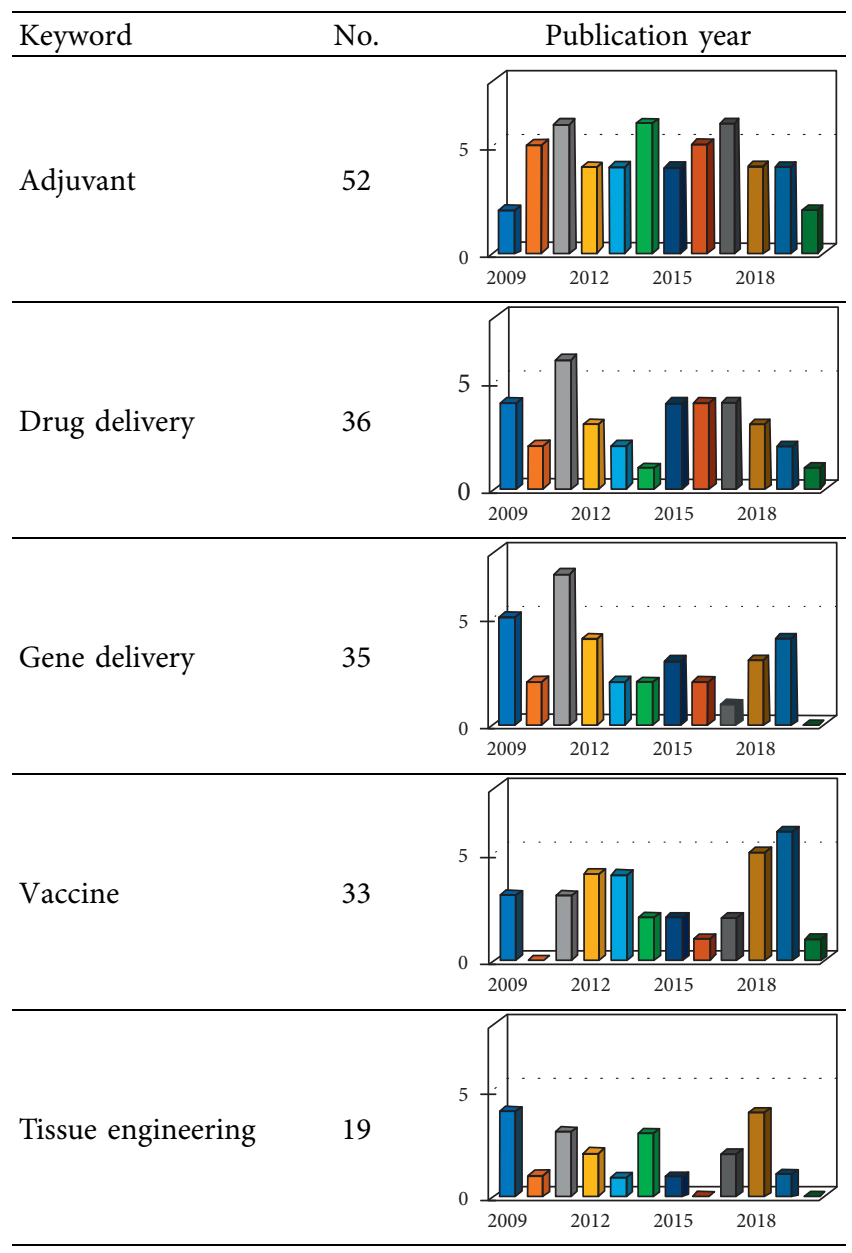

3.4. Identification of Emergent Topics. Table 3 presents the top 10 emergence scores according to the topic. Our experts concluded that most of the top 10 emergent topics are related to each other, and we ultimately categorized these 10 topics into three major emergent domains: (1) drug delivery (EScore $=20.967) ;(2)$ immune response and vaccines (EScore $=11.193$ and 8.108); and (3) polysaccharides $($ EScore $=6.908)$. In terms of the first group, drug delivery, various methods used in the preparation of CNPs include covalent cross linking, precipitation, ionic cross linking, the reverse micellar method, and emulsion-droplet coalescence. Key uses of chitosan in pharmaceutical and biomedical applications include ocular, pulmonary, nasal, and vaginal drug delivery; gene delivery; and cancer therapy [60]. Regarding group 2, Zheng et al. [61] studied the use of chitosan to treat influenza (H7N9) and found that intranasally delivered chitosan significantly enhanced the infiltration of leukocytes in the bronchoalveolar lavage. Moreover, elevated levels of proinflammatory cytokines in the bronchia revealed a potent activation of mucosal immune responses. Lastly, chitosan is emerging as the most sought-after biopolymer for use in therapeutic interventions [62].

3.5. Cross-Correlation Map of Emergence Scores and WoS Categories. Our cross-correlation analysis determined that
TABLE 3: Terms with the top 10 emergence scores.

\begin{tabular}{lcc}
\hline Emergent topics & Score & \# records \\
\hline In vitro & 46.43 & 63 \\
Drug delivery & 20.96 & 37 \\
In vivo & 14.20 & 21 \\
Immune response & 11.19 & 18 \\
Molecular weight & 8.89 & 16 \\
DNA vaccine & 8.10 & 24 \\
Delivery systems & 7.49 & 10 \\
Gene expression & 7.49 & 12 \\
Codelivery & 7.07 & 12 \\
Polysaccharide & 6.90 & 23 \\
\hline
\end{tabular}

there are 202 topics that can be automatically classified by the program into seven categories: pharmacology/pharmacy, immunology, polymer science, chemistry/multidisciplinary, biotechnology/applied microbiology, nanoscience/nanotechnology, and entomology. We found that there is cross correlation among the topics of chitosan applications in viral diseases, starting with chitosan used as a raw biopolymer material in pharmaceutical production and drug delivery related to immunology and vaccines. As identified in Figure 3, the main frontier research topics are concentrated in pharmacology/pharmacy and immunology. Interestingly, although this area is less significant to the present study, chitosan research is also found in the field of entomology, where RNA interference (RNAi) has been used for "gene silencing" via the introduction of long double-stranded RNA (dsRNA) to control pest insects [63].

3.6. Factor and Cluster Map of Emergent Topics. The 202 emergent topics can be clustered into 11 clusters (Figure 4). The lines in Figure 4 show the relationships between clusters. Strong relationships were not observed in this study, so we use dashed lines to indicate weak relationships. The clusters related to the use of chitosan in vaccine applications are the "immune response" and "vaccine delivery" clusters. These two clusters comprise the main portions in the factor map and can be interpreted as major research concentrations. However, the other clusters indicate emerging and future research frontiers. Within the two main clusters, the "immunization and trimethyl chitosan for immune response" cluster and the "DNA vaccine for vaccine clusters" can be identified as subemergent topics. According to our experts' opinion, these two clusters are related to the progress of vaccine development, and the findings are in accordance with the emergent scores presented in Table 3.

Next, we attempted a more detailed analysis of these two clusters. Table 4 shows that there are 92 articles in the immune response and vaccine delivery clusters, and the number of articles has increased over the study period. Within these clusters, the top five keywords are "vaccine," "adjuvant," "delivery," "immune response," and "induction". The table also presents the main research categories, years of publication, and the countries of the contributing researchers. The results show that the keyword "vaccine" is found in the veterinary sciences category, which indicates that a vaccine made from chitosan is under development, 


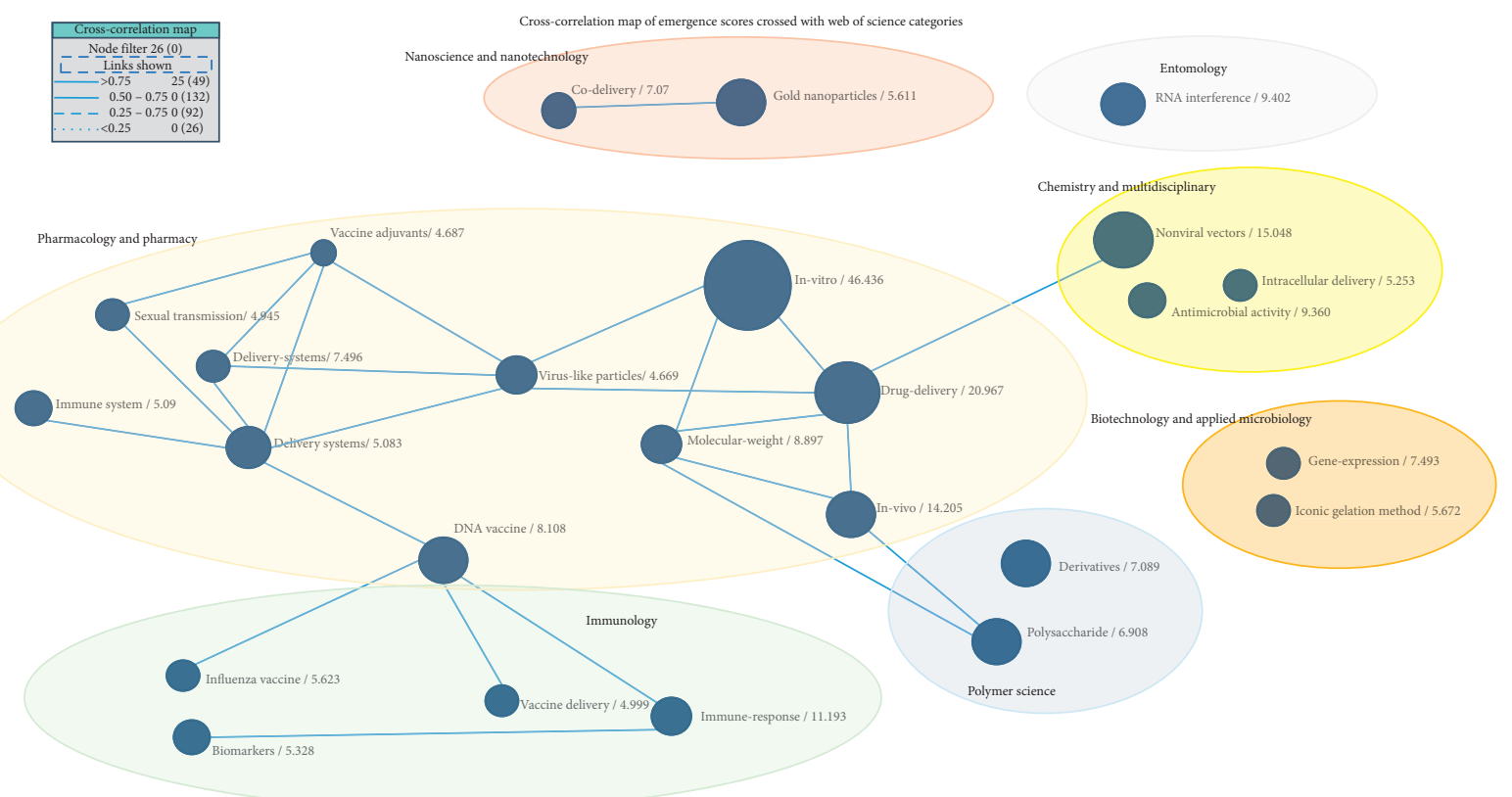

Figure 3: Cross-correlation map of emergence scores and WoS categories.

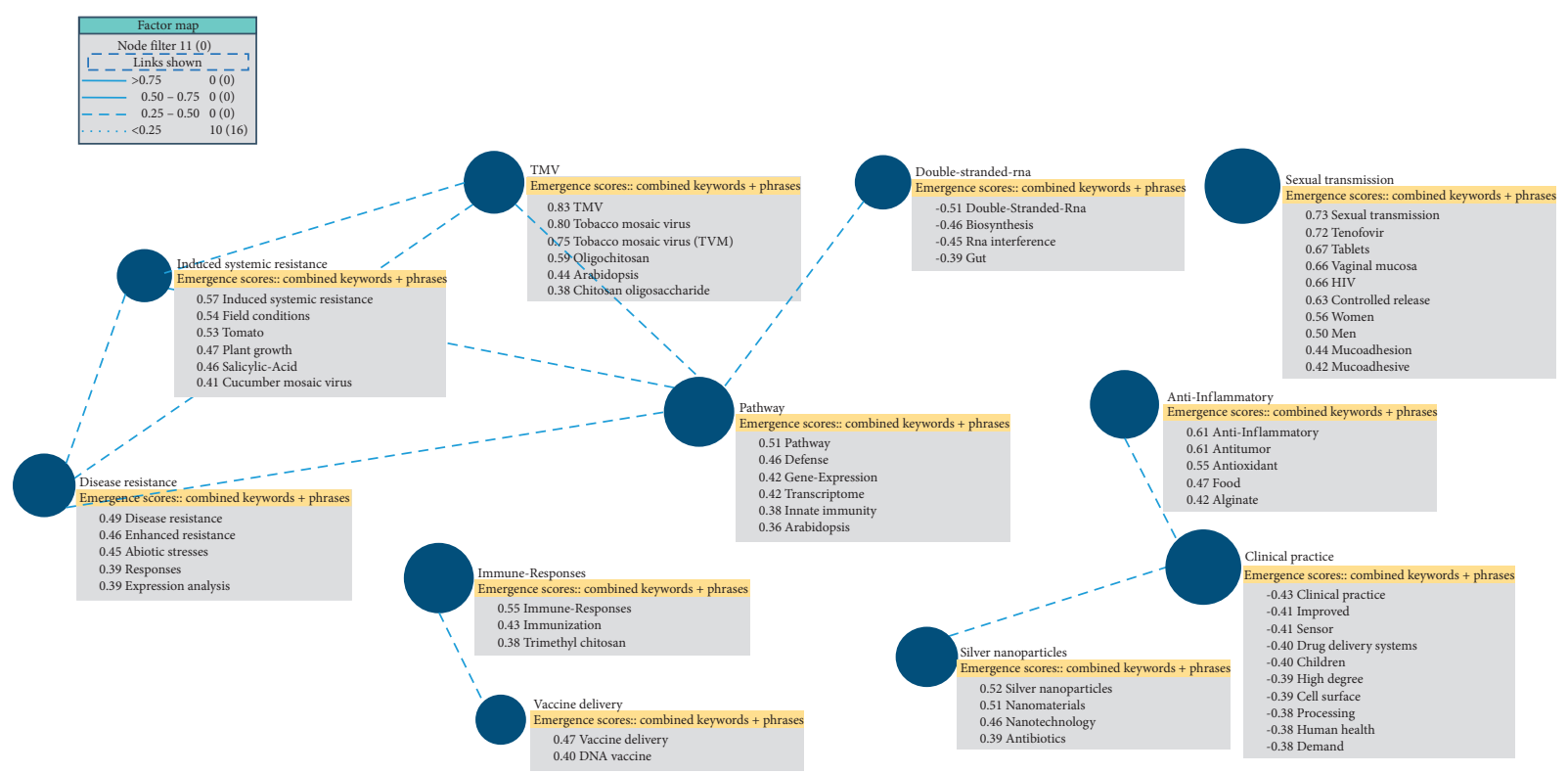

FIGURE 4: Factor map of emergence scores.

and at the time of our study, it was in the animal testing stage. For example, chitosan succinate has been applied as an adjuvant that is added to a vaccine against animal necrobacteriosis [64]. Using chitosan succinate solution as a protective medium for drying in the production of a dried inactivated vaccine against infectious bovine rhinotracheitis could preserve the biopreparation's high immunogenic activity.

Almost half of the countries that contributed to vaccinerelated publications are in Asia. China (26\%) exhibits the highest research activity, followed by South Korea (23\%). The publication trend for "vaccine" has been reasonably high after 2017. A Global Market Insights analysis indicates that increasing investment towards the development of new vaccines will drive the global vaccine market over the coming years. The projected value of the vaccine market in 2025 is up to USD 72.5 billion, which represents a significant increase from 38.5 billion in 2018. In addition, "immune response" and "delivery" (drug delivery), which are both intimately involved in vaccine development, exhibit similar publication trends [65]. China is the leader for all three keywords ("vaccine," "immune response," and "delivery"), publishing more than $20 \%$ of all articles. 
TABLE 4: Top five high-frequency keywords terms related to chitosan use in immune response and vaccine development.

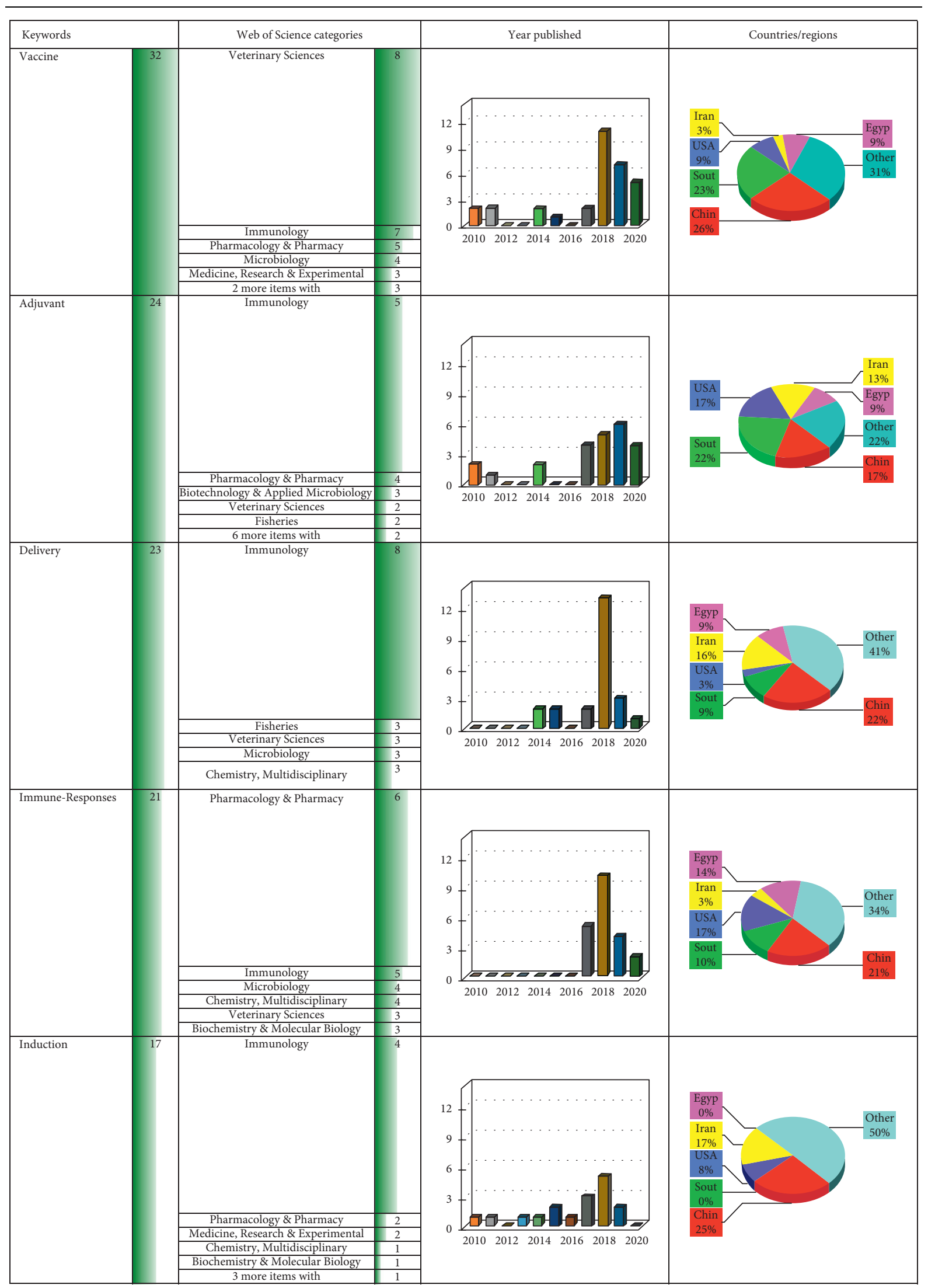




\section{Conclusions}

In this study, we adapted a process to track emerging trends in science and technology development. We also evaluated the approach using the application of chitosan in viral disease research as a test case topic. Specifically, we suggested a research approach for identifying emerging $R \& D$ areas using NLP to identify emergent indicators through a comprehensive analysis of scientific papers from the WoS.

Based on the results of step three (defining technology) and four (generating emergent scores) in the method, three potential R\&D areas were identified: drug delivery and adjuvants, vaccines and immune response, and tissue engineering. We then mapped the emergent topics to the research categories and identified two main clusters, pharmacology/pharmacy and immunology, which indicate that most research has focused on the development of chitosan for use in pharmaceuticals and immune response in beginning stage of developing. Finally, a keyword analysis for emergent topics using PCA offered possible insights for clustering developing products or services and research frontiers. The findings showed that chitosan-related research has increasingly focused on vaccine applications because major clusters were associated with the immune response and vaccine delivery. We demonstrated the predictive validity of our method by demonstrating that the emergent scores tend to increase after the 3-year base period. The quantitative results from this analysis were also checked by experts in this field to verify whether the analysis was correct from a qualitative point of view. The experts found that our results from the 1,612 WoS papers in the dataset show generalized trends and insights in terms of emergent streams, which will help researchers plan future research.

The approach presented herein could be useful for helping corporate stakeholders in technology development and manufacturing understand trends for timely exploitation. It could also help researchers and universities identify new technology pathways and determine innovative development approaches for the use of chitosan in viral disease research, thereby increasing success rates in technology commercialization and dissemination. The results of this research process could also potentially benefit readers because they would no longer need to wait for review articles, which require extensive time and energy to summarize a specific topic of interest, by which point the information would be well known and obvious for everybody working in that field. By that point, it would be too late to use such information for innovation. This process illustrates a technique to help create review articles automatically to produce accurate predictions more quickly than traditional review approaches. For example, when newcomers such as early career researchers are interested in chitosan-related research on a treatment for viral disease, they can use our findings to understand the overall research streams.

Our study findings provide guidance for understanding emerging research areas and trends in chitosan applications. The findings include the leading research countries and "hottest" topics to inform decision making by researchers at academic institutions, as well as the public and private sectors. In this analysis, we used biomedical applications of chitosan as the test model to formulate the innovative approach to explore insights; however, our approach was verified by experts in the field, indicating that it could be applied in other cases.

\section{Data Availability}

The data used to support the findings of this study are available from the corresponding author upon request.

\section{Conflicts of Interest}

The authors declare that there are no conflicts regarding this submission.

\section{Acknowledgments}

This research was supported by the Technopreneurship and Innovation Management Program, Graduate School, Chulalongkorn University, Thailand, as well as the 100th Anniversary Chulalongkorn University Fund for Doctoral Scholarship, and partially supported by the National Nanotechnology Center (NANOTEC), NSTDA, Ministry of Science and Technology, Thailand, through its program of Research Network NANOTEC (RNN). The authors thank VantagePoint (version 12.0) software from Search Technology, Inc. (http://www. theVantagePoint.com), which greatly assisted the research and helped illustrate the outcome results of this study.

\section{References}

[1] G. F. Gao, "From “A” IV to “Z”IKV: attacks from emerging and Re-emerging pathogens," Cell, vol. 172, no. 6, pp. 1157-1159, 2018.

[2] M. Woolhouse and E. Gaunt, "Ecological origins of novel human pathogens," Critical Reviews in Microbiology, vol. 33, no. 4, pp. 231-242, 2007.

[3] WHO, WHO Publishes List of Top Emerging Diseases Likely to Cause Major Epidemics, WHO, Geneva, Switzerland, 2020, https://www.who.int/medicines/ebola-treatment/WHO-listof-top-emerging-diseases/en/.

[4] C. Wang, P. W. Horby, F. G. Hayden, and G. F. Gao, "A novel coronavirus outbreak of global health concern," The Lancet, vol. 395, no. 10223, pp. 470-473, 2020.

[5] WHO, Statement on the second meeting of the International Health Regulations (2005) Emergency Committee regarding the outbreak of novel coronavirus (2019- $n \mathrm{CoV})$, WHO, Geneva, Switzerland, 2020, https://www.who.int/news-room/detail/3001-2020-statement-on-the-second-meeting-of-the-internationalhealth-regulations-(2005)-emergency-committee-regarding-theoutbreak-of-novel-coronavirus-(2019-ncov).

[6] S. Chimeno Zoth, E. Gómez, E. Carrillo, and A. Berinstein, "Locally produced mucosal IgG in chickens immunized with conventional vaccines for Newcastle disease virus," Brazilian Journal of Medical and Biological Research, vol. 41, no. 4, pp. 318-323, 2008.

[7] A. M. De Campos, Y. Diebold, E. L. S. Carvalho, A. Sánchez, and M. José Alonso, "Chitosan nanoparticles as new ocular drug delivery systems: in vitro stability, in vivo fate, and cellular toxicity," Pharmaceutical Research, vol. 21, no. 5, pp. 803-810, 2004.

[8] E. Dini, S. Alexandridou, and C. Kiparissides, "Synthesis and characterization of cross-linked chitosan microspheres for 
drug delivery applications," Journal of Microencapsulation, vol. 20 , no. 3, pp. 375-385, 2003.

[9] V. M. Correlo, L. F. Boesel, M. Bhattacharya, J. F. Mano, N. M. Neves, and R. L. Reis, "Properties of melt processed chitosan and aliphatic polyester blends," Materials Science and Engineering: A, vol. 403, no. 1-2, pp. 57-68, 2005.

[10] V. K. Mourya and N. N. Inamdar, "Chitosan-modifications and applications: opportunities galore," Reactive and Functional Polymers, vol. 68, no. 6, pp. 1013-1051, 2008.

[11] R. Jayakumar, N. Nwe, S. Tokura, and H. Tamura, "Sulfated chitin and chitosan as novel biomaterials," International Journal of Biological Macromolecules, vol. 40, no. 3, pp. 175-181, 2007.

[12] M. Rinaudo, "Chitin and chitosan: properties and applications," Progress in Polymer Science, vol. 31, no. 7, pp. 603-632, 2006.

[13] P. He, S. S. Davis, and L. Illum, "In vitro evaluation of the mucoadhesive properties of chitosan microspheres," International Journal of Pharmaceutics, vol. 166, no. 1, pp. 75-88, 1998.

[14] E. B. Denkbas and R. M. Ottenbrite, "Perspectives on: chitosan drug delivery systems based on their geometries," Journal of Bioactive and Compatible Polymers, vol. 21, no. 4, pp. 351-368, 2006.

[15] R. A. A. Muzzarelli and C. Muzzarelli, "Chitosan chemistry: relevance to the biomedical sciences," in Polysaccharides I: Structure, Characterization and Use, T. Heinze, Ed., pp. 151209, Springer Berlin Heidelberg, Berlin, Heidelberg, Germany, 2005.

[16] J. J. Wang, Z. W. Zeng, R. Z. Xiao et al., "Recent advances of chitosan nanoparticles as drug carriers," International Journal of Nanomedicine, vol. 6, pp. 765-774, 2011.

[17] M. Amidi, E. Mastrobattista, W. Jiskoot, and W. E. Hennink, "Chitosan-based delivery systems for protein therapeutics and antigens," Advanced Drug Delivery Reviews, vol. 62, no. 1, pp. 59-82, 2010.

[18] G. V. Research, "Chitosan market size, share \& trends analysis report by application (pharmaceutical \& biomedical, water treatment, cosmetics, food \& beverage), by region (APAC, North America, Europe, MEA), And Segment Forecasts," 2020, https://www.grandviewresearch.com/industry-analysis/ global-chitosan-market.

[19] B. Wilson, M. K. Samanta, K. Santhi, K. P. S. Kumar, M. Ramasamy, and B. Suresh, "Chitosan nanoparticles as a new delivery system for the anti-Alzheimer drug tacrine," Nanomedicine: Nanotechnology, Biology and Medicine, vol. 6, no. 1, pp. 144-152, 2010.

[20] H. T. Ta, H. Han, I. Larson, C. R. Dass, and D. E. Dunstan, "Chitosan-dibasic orthophosphate hydrogel: a potential drug delivery system," International Journal of Pharmaceutics, vol. 371, no. 1-2, pp. 134-141, 2009.

[21] S. Cozzens, S. Gatchair, J. Kang et al., "Emerging technologies: quantitative identification and measurement," Technology Analysis \& Strategic Management, vol. 22, no. 3, pp. 361-376, 2010.

[22] J. De Haan, "How emergence arises," Ecological Complexity, vol. 3, no. 4, pp. 293-301, 2006.

[23] J. Goldstein, "Emergence as a construct: history and issues," Emergence, vol. 1, no. 1, pp. 49-72, 1999.

[24] M. Halaweh, "Emerging technology: what is it," Journal of Technology Management \& Innovation, vol. 8, pp. 108-115, 2013.

[25] G. S. Day, P. J. H. Schoemaker, and R. E. Gunther, Wharton on Managing Emerging Technologies, John Wiley \& Sons, Hoboken, NY, USA, 2000.

[26] A. Porter, How Tech Mining Works, John Wiley \& Sons, Hoboken, NY, USA, 2004.
[27] A. L. Porter and M. J. Detampel, “Technology opportunities analysis," Technological Forecasting and Social Change, vol. 49, no. 3, pp. 237-255, 1995.

[28] R. Srinivasan, "Sources, characteristics and effects of emerging technologies: research opportunities in innovation," Industrial Marketing Management, vol. 37, no. 6, pp. 633-640, 2008.

[29] D. Rotolo, D. Hicks, and B. R. Martin, "What is an emerging technology?" Research Policy, vol. 44, no. 10, pp. 1827-1843, 2015.

[30] A. L. Porter, J. D. Roessner, X.-Y. Jin, and N. C. Newman, "Measuring national 'emerging technology' capabilities," Science and Public Policy, vol. 29, no. 3, pp. 189-200, 2002.

[31] W. Glänzel and B. Thijs, "Using 'core documents' for detecting and labelling new emerging topics," Scientometrics, vol. 91, no. 2, pp. 399-416, 2012.

[32] K. W. Boyack, R. Klavans, H. Small, and L. Ungar, "Characterizing the emergence of two nanotechnology topics using a contemporaneous global micro-model of science," Journal of Engineering and Technology Management, vol. 32, pp. 147-159, 2014.

[33] Y. Huang, J. Schuehle, A. L. Porter, and J. Youtie, "A systematic method to create search strategies for emerging technologies based on the web of science: illustrated for 'Big Data'," Scientometrics, vol. 105, no. 3, pp. 2005-2022, 2015.

[34] T.-S. Cho and H.-Y. Shih, "Patent citation network analysis of core and emerging technologies in Taiwan: 1997-2008," Scientometrics, vol. 89, no. 3, p. 795, 2011.

[35] M. N. Kyebambe, G. Cheng, Y. Huang, C. He, and Z. Zhang, "Forecasting emerging technologies: a supervised learning approach through patent analysis," Technological Forecasting and Social Change, vol. 125, pp. 236-244, 2017.

[36] S. P. Upham and H. Small, "Emerging research fronts in science and technology: patterns of new knowledge development," Scientometrics, vol. 83, no. 1, pp. 15-38, 2010.

[37] A. L. Porter, J. Garner, S. F. Carley, and N. C. Newman, "Emergence scoring to identify frontier R\&D topics and key players," Technological Forecasting and Social Change, vol. 146, pp. 628-643, 2019.

[38] B. R. Martin, "Foresight in science and technology," Technology Analysis \& Strategic Management, vol. 7, no. 2, pp. 139-168, 1995.

[39] G. S. Day and P. J. H. Schoemaker, "Avoiding the pitfalls of emerging technologies," California Management Review, vol. 42 , no. 2 , pp. 8-33, 2000.

[40] H. Small, K. W. Boyack, and R. Klavans, "Identifying emerging topics in science and technology," Research Policy, vol. 43, no. 8, pp. 1450-1467, 2014.

[41] B. C. Stahl, "What does the future hold? a critical view of emerging information and communication technologies and their social consequences," in Proceedings of the Researching the Future in Information Systems. IFIP Advances in Information and Communication Technology, pp. 59-76, Turku, Finland, June 2011.

[42] J. Alexander, J. Chase, N. Newman, A. Porter, and J. D. Roessner, "Emergence as a conceptual framework for understanding scientific and technological progress," in Proceedings of the 2012 Proceedings of PICMET 12: Technology Management for Emerging Technologies, pp. 1286-1292, Vancouver, Canada, July 2012.

[43] G. Miner, D. Delen, J. Elder, A. Fast, T. Hill, and R. A. Nisbet, "How to use the data sets and the text mining software on the companion website or on links for practical text mining," Practical Text Mining and Statistical Analysis for Non- 
structured Text Data Applications, pp. 1047-1053, Academic Press, Cambridge, MA, USA, 2012.

[44] T. P. Williams and J. Gong, "Predicting construction cost overruns using text mining, numerical data and ensemble classifiers," Automation in Construction, vol. 43, pp. 23-29, 2014.

[45] G. G. Chowdhury, "Natural language processing," Annual Review of Information Science and Technology, vol. 37, no. 1, pp. 51-89, 2003.

[46] H. Mahgoub, "Mining association rules from unstructured documents," International Journal of Applied Mathematics and Computer Sciences, vol. 1, no. 4, pp. 201-206, 2011.

[47] M. D. Khatri and S. S. Dhande, "Implementation OF text mining with auxiliary information using classification," International Journal For Technological Research In Engineering, vol. 2, no. 10, pp. 2387-2393, 2015.

[48] W. Klongthong, N. Muangsin, C. Gowanit, and V. Muangsin, "Technological convergence: the analysis of emergent topics on chitosan," in Proceedings of the ISPIM Conference, Bangkok, Thailand, March 2020.

[49] Z. Wang, A. L. Porter, X. Wang, and S. Carley, "An approach to identify emergent topics of technological convergence: a case study for 3D printing," Technological Forecasting and Social Change, vol. 146, pp. 723-732, 2019.

[50] A. L. Porter, S. W. Cunningham, J. Bank, A. T. Roper, T. W. Mason, and F. A. Rossini, Forecasting and Management of Technology, John Wiley \& Sons, Hoboken, NY, USA, 2nd edition, 2011.

[51] P.-L. Chang, C.-C. Wu, and H.-J. Leu, "Using patent analyses to monitor the technological trends in an emerging field of technology: a case of carbon nanotube field emission display," Scientometrics, vol. 82, no. 1, pp. 5-19, 2010.

[52] J. Yoon and K. Kim, "Identifying rapidly evolving technological trends for R\&D planning using SAO-based semantic patent networks," Scientometrics, vol. 88, no. 1, pp. 213-228, 2011.

[53] Y. Huang, D. Zhu, Y. Qian et al., "A hybrid method to trace technology evolution pathways: a case study of 3D printing," Scientometrics, vol. 111, no. 1, pp. 185-204, 2017.

[54] N. Altman and M. Krzywinski, "Clustering," Nature Methods, vol. 14, no. 6, pp. 545-546, 2017.

[55] J. Lever, M. Krzywinski, and N. Altman, "Principal component analysis," Nature Methods, vol. 14, no. 7, pp. 641-642, 2017.

[56] J. Li, C. Cai, J. Li et al., "Chitosan-based nanomaterials for drug delivery," Molecules, vol. 23, no. 10, 2018.

[57] A. Bernkop-Schnürch and S. Dünnhaupt, "Chitosan-based drug delivery systems," European Journal of Pharmaceutics and Biopharmaceutics, vol. 81, no. 3, pp. 463-469, 2012.

[58] S. Kole, S. S. N. Qadiri, S.-M. Shin, W.-S. Kim, J. Lee, and S.-J. Jung, "Nanoencapsulation of inactivated-viral vaccine using chitosan nanoparticles: evaluation of its protective efficacy and immune modulatory effects in olive flounder (Paralichthys olivaceus) against viral haemorrhagic septicaemia virus (VHSV) infection," Fish \& Shellfish Immunology, vol. 91, pp. 136-147, 2019.

[59] F. Croisier and C. Jérôme, "Chitosan-based biomaterials for tissue engineering," European Polymer Journal, vol. 49, no. 4, pp. 780-792, 2013.

[60] U. Garg, S. Chauhan, U. Nagaich, and N. Jain, "Current advances in chitosan nanoparticles based drug delivery and targeting," Advanced Pharmaceutical Bulletin, vol. 9, no. 2, pp. 195-204, 2019.
[61] M. Zheng, D. Qu, H. Wang et al., "Intranasal administration of chitosan against influenza A (H7N9) virus infection in a mouse model," Scientific Reports, vol. 6, no. 1, p. 28729, 2016.

[62] M. H. Periayah, A. S. Halim, and A. Z. M. Saad, "Chitosan: a promising marine polysaccharide for biomedical research," Pharmacognosy Reviews, vol. 10, no. 19, pp. 39-42, 2016.

[63] D. Ramesh Kumar, P. Saravana Kumar, M. R. Gandhi, N. A. Al-Dhabi, M. G. Paulraj, and S. Ignacimuthu, "Delivery of chitosan/dsRNA nanoparticles for silencing of wing development vestigial ( vg ) gene in Aedes aegypti mosquitoes," International Journal of Biological Macromolecules, vol. 86, pp. 89-95, 2016.

[64] A. I. Albulov, M. A. Frolova, A. V. Grin, E. I. Kovaleva, N. V. Melnik, and P. A. Krasochko, "Application of chitosan in veterinary vaccine production," Applied Biochemistry and Microbiology, vol. 54, no. 5, pp. 518-521, 2018.

[65] S. Ugalmugle and R. Swain, "Global vaccines market size by age group (pediatric, adult), by technology (conjugate, attenuated, inactivated, recombinant, toxoid), by disease (cancer, hepatitis, pneumococcal disease, DTP, dengue, influenza, human papilloma virus, meningococcal disease, polio, rotavirus), regional outlook, application potential, price trends, competitive market share \& forecast 2019-2025," 2020, https://www.gminsights.com/industry-analysis/ vaccines-market. 